\title{
Universiteit
}

Leiden

The Netherlands

\section{Dynamic effect of phase conjugation on wave localization}

\author{
Bemmel, K.J.H. van; Titov, M.L.; Beenakker, C.W.J.
}

\section{Citation}

Bemmel, K. J. H. van, Titov, M. L., \& Beenakker, C. W. J. (2002). Dynamic effect of phase conjugation on wave localization. Physical Review B, 65(17), 174203.

doi:10.1103/PhysRevB.65.174203

Version: $\quad$ Not Applicable (or Unknown)

License: $\quad$ Leiden University Non-exclusive license

Downloaded from: https://hdl.handle.net/1887/77062

Note: To cite this publication please use the final published version (if applicable). 


\title{
Dynamic effect of phase conjugation on wave localization
}

\author{
K. J. H. van Bemmel, M. Titov, and C. W. J. Beenakker \\ Instituut-Lorentz, Universiteit Leiden, P.O. Box 9506, 2300 RA Leiden, The Netherlands
}

(Received 22 October 2001; published 26 April 2002)

\begin{abstract}
We investigate what would happen to the time dependence of a pulse reflected by a disordered single-mode waveguide if it is closed at one end, not by an ordinary mirror, but by a phase-conjugating mirror. We find that the waveguide acts like a virtual cavity with resonance frequency equal to the working frequency $\omega_{0}$ of the phase-conjugating mirror. The decay in time of the average power spectrum of the reflected pulse is delayed for frequencies near $\omega_{0}$. In the presence of localization the resonance width is $\tau_{s}^{-1} \exp (-L / l)$, with $L$ the length of the waveguide, $l$ the mean free path, and $\tau_{s}$ the scattering time. Inside this frequency range the decay of the average power spectrum is delayed up to times $t \simeq \tau_{s} \exp (L / l)$.
\end{abstract}

DOI: 10.1103/PhysRevB.65.174203

PACS number(s): 42.65.Hw, 42.25.Dd, 72.15.Rn

\section{INTRODUCTION}

The reflection of a wave pulse by a random medium provides insight into the dynamics of localization. ${ }^{1-4}$ The reflected amplitude contains rapid fluctuations over a broad range of frequencies, with a slowly decaying envelope. The power spectrum $a(\omega, t)$ characterizes the decay in time $t$ of the envelope at frequency $\omega$. In an infinitely long waveguide (with $N$ propagating modes), the signature of localization, 5,6

$$
\langle a(\omega, t)\rangle \propto t^{-2} \text { for } t \gg N^{2} \tau_{s},
$$

is a quadratic decay of the disorder-averaged power spectrum $\langle a\rangle$, which sets in after $N^{2}$ scattering times $\tau_{s}$.

The decay (1) still holds over a broad range of times if the length $L$ of the waveguide is finite, but much greater than the localization length $\xi=(N+1) l$ (with $l=c \tau_{s}$ the mean free path). What changes is that for exponentially large times $t$ $\gg \tau_{s} \exp (L / l)$ the quadratic decay becomes more rapid $\propto \exp \left(-\right.$ const $\left.\times \ln ^{2} t\right)$. This is the celebrated log-normal tail. $^{t-11}$ We may assume that the finite length of the waveguide is realized by terminating one end by a perfectly reflecting mirror, so that the total reflected power is unchanged.

In this paper we ask the question what happens if instead of such a normal mirror one would use a phase-conjugating mirror. ${ }^{12,13}$ The interplay of multiple scattering by disorder and optical phase conjugation is a rich problem even in the static case. ${ }^{14-16}$ Here we show that the dynamical aspects are particularly striking. Basically, the disordered waveguide is turned into a virtual cavity with a resonance frequency $\omega_{0}$ set by the phase-conjugating mirror.

We present a detailed analytical and numerical calculation for the single-mode case $(N=1)$. For times $t \gg \tau_{s}$ we find that $a(\omega, t)$ has decayed almost completely except in a narrow frequency range $\propto \tau_{s}^{-1} \exp (-L / l)$ around $\omega_{0}$. In this frequency range the decay is delayed up to times $t$ $\simeq \tau_{s} \exp (L / l)$, after which a log-normal decay sets in. The exponentially large difference in time scales for the decay near $\omega_{0}$ and away from $\omega_{0}$ is a signature of localization.

\section{FORMULATION OF THE PROBLEM}

\section{A. Scattering theory}

A scattering matrix formulation of the problem of combined elastic scattering by disorder and inelastic scattering by a phase-conjugating mirror was developed by Paasschens et al. ${ }^{15}$ We summarize the basic equations for the case of a single propagating mode in the geometry shown in Fig. 1. A single-mode waveguide is closed at one end $(x=0)$ by either a normal mirror or by a phase-conjugating mirror. Elastic scattering in the waveguide is due to random disorder in the region $0<x<L$. For simplicity we consider a single polarization, so that we can use a scalar wave equation.

The phase-conjugating mirror consists of a four-wave mixing cell: ${ }^{12,13}$ Two counterpropagating beams at frequency $\omega_{0}$ mix with an incident beam at frequency $\omega_{0}+\omega$ to yield a retroreflected beam at frequency $\omega_{0}-\omega$ (for $\omega \ll \omega_{0}$ ). The mixing is due to the presence in the cell of a medium with a large third-order nonlinear susceptibility (e.g., $\mathrm{BaTiO}_{3}$ or $\mathrm{CS}_{2}$ ).

For $x \gg L$ the wave amplitude at frequencies $\omega_{ \pm}=\omega_{0}$ $\pm \omega$ is an incoming or outgoing plane wave,

$$
\begin{aligned}
& u_{ \pm}^{\text {in }}(\vec{r}, t)=\operatorname{Re} \phi_{ \pm}^{\text {in }} \exp \left[-i k_{ \pm}(x-L)-i \omega_{ \pm} t\right] \psi_{ \pm}(y, z), \\
& u_{ \pm}^{\text {out }}(\vec{r}, t)=\operatorname{Re} \phi_{ \pm}^{\text {out }} \exp \left[i k_{ \pm}(x-L)-i \omega_{ \pm} t\right] \psi_{ \pm}(y, z) .
\end{aligned}
$$

Here $k_{ \pm}=k_{0} \pm \omega / c$ is the wave number at frequency $\omega_{ \pm}$, with $k_{0}$ the wave number at $\omega_{0}$ and $c=d \omega / d k$ the group velocity. The transverse wave profile $\psi_{ \pm}(y, z)$ is normalized such that the wave carries unit flux.

The reflection matrix relates the incoming and outgoing wave amplitudes, according to

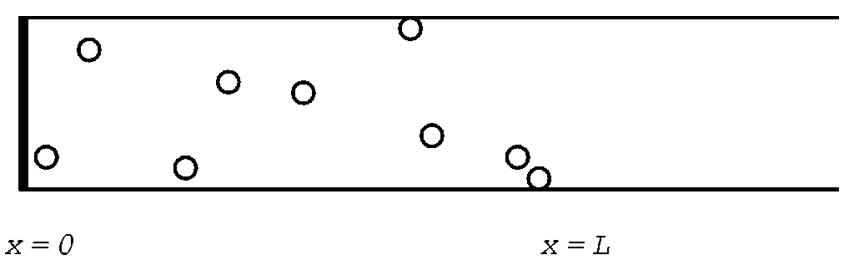

FIG. 1. The geometry under investigation consists of a singlemode waveguide with a mirror at $x=0$. It can be a normal mirror or a phase-conjugating mirror. There are randomly positioned obstacles between $x=0$ and $x=L$. 


$$
\left(\begin{array}{c}
\phi_{+} \\
\phi_{-}^{*}
\end{array}\right)^{\text {out }}=\left(\begin{array}{ll}
r_{++} & r_{+-} \\
r_{-+} & r_{--}
\end{array}\right)\left(\begin{array}{c}
\phi_{+} \\
\phi_{-}^{*}
\end{array}\right)^{\text {in }} .
$$

The reflection coefficients are complex numbers that depend on $\omega$. They satisfy the symmetry relations

$$
r_{--}^{*}(\omega)=r_{++}(-\omega), \quad r_{-+}^{*}(\omega)=r_{+-}(-\omega) .
$$

If there is only reflection at the mirror and no disorder, then one has simply

$$
\left(\begin{array}{ll}
r_{++} & r_{+-} \\
r_{-+} & r_{--}
\end{array}\right)=\left(\begin{array}{cc}
-e^{2 i k_{+} L} & 0 \\
0 & -e^{-2 i k_{-} L}
\end{array}\right)
$$

for a normal mirror and

$$
\left(\begin{array}{ll}
r_{++} & r_{+-} \\
r_{-+} & r_{--}
\end{array}\right)=\left(\begin{array}{cc}
0 & -i e^{2 i L \omega / c} \\
i e^{2 i L \omega / c} & 0
\end{array}\right)
$$

for a phase-conjugating mirror operating in the regime of ideal retroreflection. (We will assume this regime in what follows.)

We wish to determine how the reflection coefficients are modified by the elastic scattering by the disorder. For this we need the elastic scattering matrix

$$
S=\left(\begin{array}{ll}
r & t^{\prime} \\
t & r^{\prime}
\end{array}\right) .
$$

The reflection coefficients $r, r^{\prime}$ and transmission coefficients $t, t^{\prime}$ describe reflection and transmission from the left or from the right of a segment of a disordered waveguide of length $L$. The matrix $S$ is unitary and symmetric (hence $t=t^{\prime}$ ). The basis for $S$ is chosen such that $r=r^{\prime}=0, t( \pm \omega)=e^{i k_{ \pm} L}$ in the absence of disorder. The relationship between the coefficients in Eqs. (3) and (7) is ${ }^{15}$

$$
\begin{gathered}
r_{++}(\omega)=r^{\prime}(\omega)+t(\omega)\left[1-r^{*}(-\omega) r(\omega)\right]^{-1} r^{*}(-\omega) t(\omega), \\
r_{+-}(\omega)=-i t(\omega)\left[1-r^{*}(-\omega) r(\omega)\right]^{-1} t^{*}(-\omega),
\end{gathered}
$$

for a phase-conjugating mirror. For a normal mirror there is no mixing of frequencies and one has simply

$$
\begin{gathered}
r_{++}(\omega)=r^{\prime}(\omega)-t(\omega)[1+r(\omega)]^{-1} t(\omega), \\
r_{+-}(\omega)=0 .
\end{gathered}
$$

In each case the matrix of reflection coefficients is unitary, so

$$
\left|r_{++}(\omega)\right|^{2}+\left|r_{+-}(\omega)\right|^{2}=1 .
$$

\section{B. Power spectrum}

We assume that a pulse $\propto \delta(t)$ is incident at $x=L$ [corresponding to $\phi_{ \pm}^{\text {in }}=1$ for all $\omega$ in Eq. (2)]. The reflected wave at $x=L$ has amplitude

$$
\begin{aligned}
u_{\mathrm{out}}(t)= & \operatorname{Re} e^{-i \omega_{0} t} \int_{0}^{\infty} \frac{d \omega}{2 \pi}\left\{\left[r_{++}(\omega)+r_{+-}(\omega)\right] e^{-i \omega t}\right. \\
& \left.+\left[r_{--}^{*}(\omega)+r_{-+}^{*}(\omega)\right] e^{i \omega t}\right\} .
\end{aligned}
$$

(We have suppressed the transverse coordinates $y, z$ for simplicity of notation.) Using the symmetry relations (4), we can rewrite this as

$$
u_{\text {out }}(t)=\operatorname{Re} e^{-i \omega_{0} t} \int_{-\infty}^{\infty} \frac{d \omega}{2 \pi}\left[r_{++}(\omega)+r_{+-}(\omega)\right] e^{-i \omega t} .
$$

The time correlator of the reflected wave becomes

$$
\begin{aligned}
u_{\mathrm{out}}(t) & u_{\mathrm{out}}\left(t+t^{\prime}\right) \\
= & \frac{1}{2} \operatorname{Re} e^{i \omega_{0} t^{\prime}} \int_{-\infty}^{\infty} \frac{d \omega}{2 \pi} \int_{-\infty}^{\infty} \frac{d \omega^{\prime}}{2 \pi} e^{i\left(\omega^{\prime}-\omega\right) t} e^{i \omega^{\prime} t^{\prime}} \\
& \times\left[r_{++}(\omega)+r_{+-}(\omega)\right]\left[r_{++}^{*}\left(\omega^{\prime}\right)+r_{+-}^{*}\left(\omega^{\prime}\right)\right],
\end{aligned}
$$

plus terms that oscillate on a time scale $1 / \omega_{0}$. We make the rotating wave approximation and neglect these rapidly oscillating terms. The power spectrum $a$ of the reflected wave is obtained by a Fourier transform

$$
\begin{aligned}
a(\omega, t) & =\int_{-\infty}^{\infty} d t^{\prime} \cos \left[\left(\omega_{0}+\omega\right) t^{\prime}\right] u_{\text {out }}(t) u_{\text {out }}\left(t+t^{\prime}\right) \\
& =\operatorname{Re} \int_{-\infty}^{\infty} \frac{d \delta \omega}{2 \pi} e^{-i \delta \omega t} a(\omega, \delta \omega),
\end{aligned}
$$

where we have introduced the correlator in the frequency domain

$$
\begin{aligned}
a(\omega, \delta \omega)= & \frac{1}{4}\left[r_{++}(\omega+\delta \omega)+r_{+-}(\omega+\delta \omega)\right]\left[r_{++}^{*}(\omega)\right. \\
& \left.+r_{+-}^{*}(\omega)\right] .
\end{aligned}
$$

Integration of the power spectrum over time yields, using also Eq. (10),

$$
\begin{aligned}
\int_{-\infty}^{\infty} d t a(\omega, t) & =\operatorname{Re} a(\omega, \delta \omega=0) \\
& =\frac{1}{4}+\frac{1}{2} \operatorname{Re} r_{+-}(\omega) r_{++}^{*}(\omega) .
\end{aligned}
$$

For a normal mirror $r_{+-}(\omega)=0$ and $a(\omega, \delta \omega=0)=\frac{1}{4}$, expressing flux conservation. For the phase-conjugating mirror there is inelastic scattering, which mixes the frequency components $\omega$ and $-\omega$. The constraint of flux conservation then takes the form

$$
a(\omega, \delta \omega=0)+a(-\omega, \delta \omega=0)=\frac{1}{2} .
$$

This follows from the symmetry relations (4) and the unitarity of the reflection matrix. Equation (17) implies that $a(\omega$ $=0, \delta \omega=0)=\frac{1}{4}$. 


\section{RANDOM SCATTERERS}

We assume weak disorder, meaning that the mean free path $l$ is much larger than the wavelength $2 \pi / k_{0}$. The multiple scattering by disorder localizes the wave with localization length equal to $2 l$. We consider separately the case of a phase-conjugating mirror and of a normal mirror.

\section{A. Phase-conjugating mirror}

We first concentrate on the degenerate regime of small frequency shift $\omega$ and will simplify the expressions by putting $\omega=0$ from the start. We note that $r_{++}(0)=0, r_{+-}(0)$ $=-i$, as follows from Eq. (8) and unitarity of the scattering matrix (7). Using Eqs. (8) and (15), we arrive at the power spectrum in the frequency domain

$$
\begin{aligned}
a(0, \delta \omega)= & \frac{i}{4}\left\{r^{\prime}(\delta \omega)+\left[1-r^{*}(-\delta \omega) r(\delta \omega)\right]^{-1}\right. \\
& \left.\times\left[t^{2}(\delta \omega) r^{*}(-\delta \omega)-i t(\delta \omega) t^{*}(-\delta \omega)\right]\right\} .
\end{aligned}
$$

The scattering amplitudes have the polar decomposition $r$ $=\sqrt{R} \exp (i \theta), \quad r^{\prime}=\sqrt{R} \exp \left(i \theta^{\prime}\right), \quad$ and $\quad t=i \sqrt{1-R} \exp \left[\frac{1}{2} i(\theta\right.$ $\left.\left.+\theta^{\prime}\right)\right]$, with $R, \theta, \theta^{\prime}$ real functions of frequency. The phase $\theta^{\prime}$ may be assumed to be statistically independent of $R$ $( \pm \delta \omega), \theta( \pm \delta \omega)$, and uniformly distributed in $(0,2 \pi)$. (This is the Wigner conjecture, proven for chaotic scattering in Ref. 17.) In this way only the last term in Eq. (18) survives the disorder average $\langle\cdots\rangle$,

$$
4\langle a(0, \delta \omega)\rangle=\left\langle\frac{t(\delta \omega) t^{*}(-\delta \omega)}{1-r^{*}(-\delta \omega) r(\delta \omega)}\right\rangle=\sum_{m=0}^{\infty} Z_{m},
$$

where we have defined $Z_{m}=\left\langle t(\delta \omega) t^{*}(-\delta \omega)\right.$ $\left.\times\left[r^{*}(-\delta \omega) r(\delta \omega)\right]^{m}\right\rangle$.

The moments $Z_{m}$ satisfy the Berezinskii recursion relation $^{18,19}$

$$
\begin{aligned}
l \frac{\mathrm{d} Z_{m}}{\mathrm{~d} L}= & m^{2}\left(Z_{m+1}+Z_{m-1}-2 Z_{m}\right)+(2 m+1)\left(Z_{m+1}-Z_{m}\right) \\
& +2 i \tau_{s} \delta \omega(2 m+1) Z_{m}
\end{aligned}
$$

with $\tau_{s}=l / c$ the scattering time. (The mean free path $l$ accounts only for backscattering, so that the scattering time in a kinetic equation would equal $\frac{1}{2} \tau_{s}$.) The initial condition is $Z_{m}(L=0)=\delta_{m, 0}$. In Appendix A we derive an analytical result for $\langle a(0, \delta \omega)\rangle$ in the small frequency range $\ln \left(1 / \tau_{s} \delta \omega\right)$ $\gtrsim L / l \gg 1$. It reads

$$
\begin{aligned}
\langle a(0, \delta \omega)\rangle= & \frac{1}{2} \int_{-\infty}^{\infty} d k i k\left(-2 i \tau_{s} \delta \omega\right)^{i k-1 / 2} 2^{-3 i k-1 / 2} \\
& \times \Gamma^{2}\left(\frac{1}{2}+i k\right) \Gamma\left(\frac{1}{2}-i k\right) \Gamma^{-1}(1+i k) \Gamma^{-1}(i k) \\
& \times \exp \left[-\left(\frac{1}{4}+k^{2}\right) L / l\right] .
\end{aligned}
$$

The initial decay is determined by the contributions of the poles at $k=-\frac{1}{2} i,-\frac{3}{2} i,-\frac{5}{2} i$,
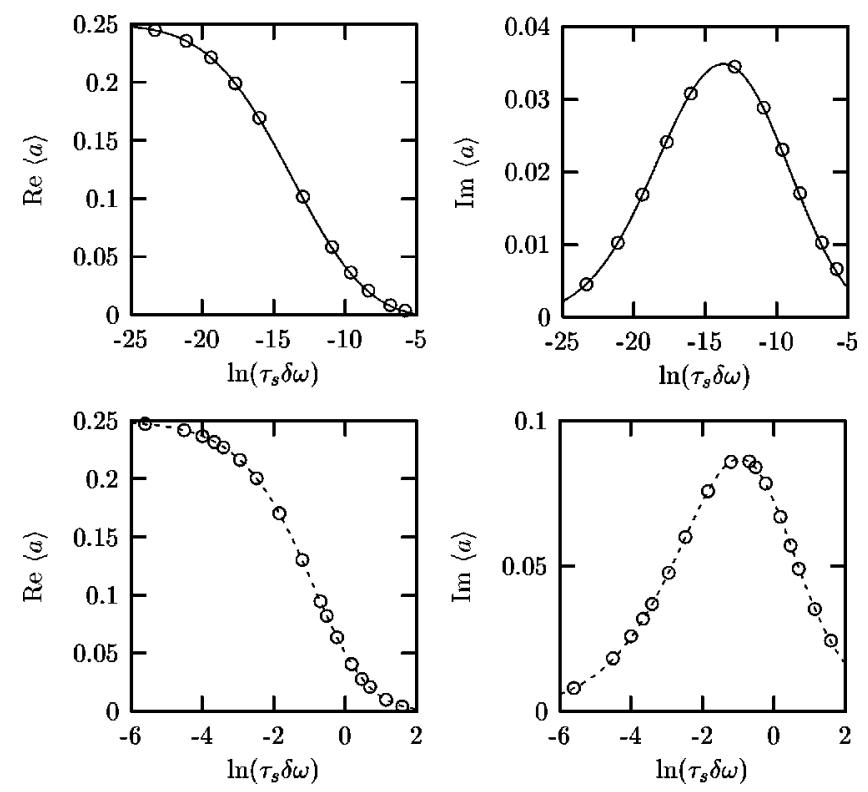

FIG. 2. Average power spectrum for reflection by a disordered waveguide $(L / l=12.3)$ connected to a phase-conjugating mirror [solid curves, from Eq. (21)] or a normal mirror [dashed curves, from Eq. (28)]. The data points follow from a numerical simulation. There is no adjustable parameter in the comparison. Notice the much faster frequency dependence for the phase-conjugating mirror (top panels), compared to the normal mirror (bottom panels).

$$
\begin{aligned}
\langle a(0, \delta \omega)\rangle= & \frac{1}{4}+\frac{1}{4} i \tau_{s} \delta \omega \exp (2 L / l)-\frac{1}{18} \tau_{s}^{2} \delta \omega^{2} \exp (6 L / l) \\
& +\mathrm{O}\left(\delta \omega^{3}\right)
\end{aligned}
$$

The result $(21)$ is plotted in Fig. 2 for $L / l=12.3$. We compare with the data from a numerical solution of the wave equation on a two-dimensional lattice, using the method of recursive Green functions. ${ }^{20}$ (The method of simulation is the same as in Ref. 15, and we refer to that paper for a more detailed description.) The agreement with the analytical curves is quite good, without any adjustable parameter. The $\delta \omega$ dependence of $\langle a(0, \delta \omega)\rangle$ for large $L / l$ occurs on an exponentially small scale, within the range of validity of Eq. (21).

A Fourier transform of Eq. (21) yields the average power spectrum in the time domain for $\ln \left(t / \tau_{s}\right) \gg L / l \gg 1$, with the result

$$
\begin{aligned}
\langle a(0, t)\rangle= & \frac{1}{8} \pi^{3 / 2}(L / l)^{-3 / 2} \exp (-L / 4 l) \tau_{s}^{-1 / 2} t^{-1 / 2} \\
& \times \ln \left(4 t / \tau_{s}\right) \exp \left[-(l / 4 L) \ln ^{2}\left(4 t / \tau_{s}\right)\right] .
\end{aligned}
$$

The leading logarithmic asymptote of the decay is lognormal, $\propto \exp \left[-(l / 4 L) \ln ^{2} t\right]$, characteristic of anomalously localized states. $^{7-11}$

These results are calculated for $\omega=0$ and remain valid as long as $\omega \ll \tau_{s}^{-1} \exp (-L / l)$. This can be checked by performing a Taylor expansion in $\omega$ of Eq. (8), using the polar decomposition for $r, r^{\prime}, t$. We still have $r_{++}(\omega) \approx 0$ and $r_{+-}(\omega) \approx-i$ as long as $\omega d \theta / d \omega \ll 1-R$. In order of magnitude this corresponds to $\tau_{s} \omega \ll \exp (-L / l)$. This is the degenerate regime. For $\tau_{s} \omega \gg \exp (-L / l)$ the power spectrum 
$a(\omega, \delta \omega)$ is dominated by the term $r^{\prime}(\omega+\delta \omega) r^{\prime *}(\omega)$. The decay of $\langle a(\omega, \delta \omega)\rangle$ then occurs in the range $\tau_{s} \delta \omega \lesssim 1$. The same is true for the normal mirror, which we consider in the next subsection. The presence of the mirror is now only of importance for very small $\delta \omega\left[\ln \left(1 / \tau_{s} \delta \omega\right) \geq L / l \gg 1\right]$, when $a(\omega, \delta \omega) \approx \frac{1}{4}$. For $\tau_{s} \omega \gg 1$ the average power spectrum $\langle a(\omega, \delta \omega)\rangle$ in the range $\ln \left(1 / \tau_{s} \delta \omega\right) \gg L / l$ is the same as that for a normal mirror, leading to exactly the same log-normal decay in the time domain. This is proven in Appendix B.

\section{B. Normal mirror}

For comparison we discuss the known results for a disordered waveguide connected to a normal mirror instead of a phase-conjugating mirror. Since $r_{+-}=0$, one has from Eq. (15)

$$
4\langle a(\omega, \delta \omega)\rangle=\left\langle r_{++}(\omega+\delta \omega) r_{++}^{*}(\omega)\right\rangle \equiv R_{1} .
$$

The quantities $R_{m}=\left\langle\left[r_{++}(\omega+\delta \omega) r_{++}^{*}(\omega)\right]^{m}\right\rangle$ satisfy the Berezinskii recursion relation ${ }^{18,19}$

$$
l \frac{d R_{m}}{d L}=m^{2}\left(R_{m+1}+R_{m-1}-2 R_{m}\right)+2 i \tau_{s} \delta \omega m R_{m} .
$$

The initial condition is $R_{m}(L=0)=1$ for all $m$. The solution for $\ln \left(1 / \tau_{s} \delta \omega\right) \geq L / l$ is known ${ }^{21}$ and gives the average power spectrum

$$
\begin{aligned}
\langle a(\omega, \delta \omega)\rangle= & \frac{1}{2} \sqrt{-2 i \tau_{s} \delta \omega}\left(\mathrm{K}_{1}\left[2 \sqrt{-2 i \tau_{s} \delta \omega}\right]\right. \\
& +\frac{1}{\pi} \int_{-\infty}^{\infty} d k k \sinh (\pi k)\left(\frac{1}{4}+k^{2}\right)^{-1} \\
& \left.\times \mathrm{K}_{2 i k}\left[2 \sqrt{-2 i \tau_{s} \delta \omega}\right] \exp \left[-\left(\frac{1}{4}+k^{2}\right) L / l\right]\right),
\end{aligned}
$$

with $\mathrm{K}$ a Bessel function. [The result (26) does not require $L / l \gg 1$, in contrast to Eq. (21).] The initial decay is dominated by the contributions of the poles at $k=-\frac{1}{2} i,-\frac{3}{2} i$, $-\frac{5}{2} i$,

$\langle a(\omega, \delta \omega)\rangle=\frac{1}{4}+\frac{1}{2} i \tau_{s} \delta \omega L / l-\frac{1}{4} \tau_{s}^{2} \delta \omega^{2} \exp (2 L / l)+O\left(\delta \omega^{3}\right)$.

Comparison of Eqs. (26) and (27) with Eqs. (21) and (22) shows that the decay is much slower for a normal mirror than for a phase-conjugating mirror. The characteristic frequency scale is larger by a factor $\exp (2 L / l)$. So Eq. (26) is not sufficient to describe the entire decay of $\langle a(\omega, \delta \omega)\rangle$, which occurs in the range $\tau_{s} \delta \omega \lesssim 1$. The decay in this range is obtained by putting the left-hand side of Eq. (25) equal to zero, leading to ${ }^{5,22}$

$$
\langle a(\omega, \delta \omega)\rangle=\frac{1}{4}-\frac{1}{2} i \tau_{s} \delta \omega \exp \left(-2 i \tau_{s} \delta \omega\right) \operatorname{Ei}\left(2 i \tau_{s} \delta \omega\right),
$$

where $\mathrm{Ei}$ is the exponential integral function. The range of validity of Eq. (28) is $\ln \left(1 / \tau_{s} \delta \omega\right) \ll L / l$ and $L / l \gg 1$. The re- sult (28) is plotted in Fig. 2 and is seen to agree well with data from the numerical simulation.

For $\ln \left(t / \tau_{s}\right) \ll L / l$ (and $L / l \gg 1$ ) one can perform the Fourier transform of Eq. (28) to get the average power spectrum in the time domain ${ }^{5}$

$$
\langle a(\omega, t)\rangle=\frac{1}{2} \tau_{s}\left(t+2 \tau_{s}\right)^{-2}, \quad t>0 .
$$

It decays quadratically $\propto t^{-2}$ for $t / \tau_{s} \gg 1$. For exponentially long times $t \gg \tau_{s} \exp (L / l)$, one should instead perform the Fourier transform of Eq. (26). One finds that the quadratic decay crosses over to a log-normal decay $\propto \exp$ $\left[-(l / 4 L) \ln ^{2} t\right],{ }^{7}$ the same as for the phase-conjugating mirror.

\section{CONCLUSION}

We have shown that the interplay of phase conjugation and multiple scattering by disorder leads to a drastic slowing down of the decay in time $t$ of the average power spectrum $\langle a(\omega, t)\rangle$ of frequency components $\omega$ of a reflected pulse. The slowing down exists in a narrow frequency range around the characteristic frequency $\omega_{0}$ of the phase-conjugating mirror (degenerate regime). If $\omega$ is outside this frequency range (nondegenerate regime), the power spectrum decays as rapidly as for a normal mirror.

The slowing down can be interpreted in terms of a longlived resonance at $\omega_{0}$, which is induced in the random medium by the phase-conjugating mirror. This resonance is known from investigations of the static scattering properties. ${ }^{15}$ The resonance is exponentially narrow, $\propto \tau_{s}^{-1} \exp (-L / l)$, in the presence of localization (with $\tau_{s}$ the scattering time, $L$ the length of the disordered region, and $l$ the mean free path). The resonance leads to the exponentially large differences in time scales for the decay of the power spectrum in the degenerate regime and the nondegenerate regime.

We have restricted the calculation in this paper to the case of a single propagating mode, when a complete analytical theory could be provided. We expect that the $N$-mode case is qualitatively similar: An exponentially large difference in time scales $\propto \exp (L / \xi)$ for the decay in the degenerate and nondegenerate regimes provided the medium is localized $[L$ large compared to the localization length $\xi=(N+1) l]$. In the diffusive regime we expect $\langle a(\omega, t)\rangle$ to decay on the time scale of the diffusion time $\tau_{s}(L / l)^{2}$. The difference with the nondegenerate regime (or a normal mirror) is then a factor $(L / l)^{2}$ instead of exponentially large.

In final analysis we see that phase conjugation greatly magnifies the difference in the dynamics with and without localization. Indeed, if there is no phase-conjugating mirror the main difference is a decay $\propto t^{-3 / 2}$ in the diffusive regime versus $t^{-2}$ in the localized regime, ${ }^{6}$ but the characteristic time scale remains the same (set by the scattering time $\tau_{s}$ ). We therefore suggest that phase conjugation might be a promising tool in the ongoing experimental search for dynamical features of localization. ${ }^{23,24}$ 


\section{APPENDIX A: POWER SPECTRUM IN THE FREQUENCY DOMAIN}

We show how to arrive at the result (21) starting from the recursion relation $(20)$. We assume $\ln \left(1 / \tau_{s} \delta \omega\right) \gtrsim L / l \gg 1$. It is convenient to work with the Laplace transform

$$
Z_{m}(\lambda)=\int_{0}^{\infty} \frac{d L}{l} \exp (-\lambda L / l) Z_{m}(L)
$$

of the moments $Z_{m}$. The recursion relation (20) transforms into

$$
\begin{aligned}
\lambda Z_{m}(\lambda)-\delta_{m, 0}= & m^{2}\left[Z_{m+1}(\lambda)+Z_{m-1}(\lambda)-2 Z_{m}(\lambda)\right] \\
& +(2 m+1)\left[Z_{m+1}(\lambda)-Z_{m}(\lambda)\right] \\
& -\beta(2 m+1) Z_{m}(\lambda),
\end{aligned}
$$

with $\beta=-2 i \tau_{s} \delta \omega$.

For small $|\beta|$ and large $m$ this equation can be written as a differential equation

$$
m^{2} \frac{\partial^{2} Z(m, \lambda)}{\partial m^{2}}+2 m \frac{\partial Z(m, \lambda)}{\partial m}-(\lambda+2 \beta m) Z(m, \lambda)=0
$$

where $m$ is now considered to be a continuous variable. The solution of Eq. (A3) is

$$
Z(m, \lambda)=C(\lambda, \beta)(\beta m)^{-1 / 2} \mathrm{~K}_{\sqrt{1+4 \lambda}}(2 \sqrt{2 \beta m})
$$

The factor $C(\lambda, \beta)$ is determined by matching to the solution of Eq. (A2) for $\beta m \rightarrow 0, m \rightarrow \infty$, which has been calculated in Ref. 25. The result is

$$
\begin{aligned}
C(\lambda, \beta)= & 4 \pi \beta^{1 / 2} \Gamma\left(\frac{1}{2}+\frac{1}{2} \sqrt{1+4 \lambda}\right) \\
& \times \Gamma^{-1}\left(1+\frac{1}{2} \sqrt{1+4 \lambda}\right) \Gamma^{-1}\left(\frac{1}{2} \sqrt{1+4 \lambda}\right) \\
& \times \exp \left[\frac{1}{2} \sqrt{1+4 \lambda} \ln (\beta / 8)\right] .
\end{aligned}
$$

To obtain the power spectrum (19) we replace the sum over $m$ by an integration, with the result

$$
\begin{aligned}
\sum_{m=0}^{\infty} Z_{m}(\lambda)= & 2^{1 / 2} \pi \beta^{-1 / 2} \Gamma^{2}\left(\frac{1}{2}+\frac{1}{2} \sqrt{1+4 \lambda}\right) \\
& \times \Gamma\left(\frac{1}{2}-\frac{1}{2} \sqrt{1+4 \lambda}\right) \Gamma^{-1}(1 \\
& \left.+\frac{1}{2} \sqrt{1+4 \lambda}\right) \Gamma^{-1}\left(\frac{1}{2} \sqrt{1+4 \lambda}\right) \\
& \times \exp \left[\frac{1}{2} \sqrt{1+4 \lambda} \ln (\beta / 8)\right] .
\end{aligned}
$$

There are poles at $\lambda=n(n+1), n=0,1,2, \ldots$, and a branch cut starting at $\lambda=-1 / 4$. When doing the inverse Laplace transform we put the integration path in between the poles and the branch cut. The final result is given by Eq. (21). The reason that we need the condition $L / l \gg 1$ is that Eqs. (A4) and (A5) are only correct for $m \gg 1$. The first terms in the sum $\sum_{m=0}^{\infty} Z_{m}$ are important for $L / l \leqq 1$, but can be neglected for $L / l \gg 1$.

\section{APPENDIX B: EQUIVALENCE OF NORMAL AND PHASE-CONJUGATING MIRROR IN THE NONDEGENERATE REGIME}

We show that the average power spectrum $\langle a(\omega, \delta \omega)\rangle$ in the range $\ln \left(1 / \tau_{s} \delta \omega\right) \gg L / l$ is the same for a normal mirror and a phase-conjugating mirror in the regime $\tau_{s} \omega \gg 1$.

First we consider the normal mirror. One can write $\langle a(\omega, \delta \omega)\rangle$ in terms of $R, \theta, \theta^{\prime}$, using the polar decomposition and Eqs. (9) and (15). Only two terms survive the average over $\theta$,

$$
4\langle a(\omega, \delta \omega)\rangle=\left\langle r^{\prime}(\omega+\delta \omega) r^{\prime *}(\omega)\right\rangle+\left\langle\frac{t^{2}(\omega+\delta \omega) t^{2 *}(\omega)}{[1+r(\omega+\delta \omega)]\left[1+r^{*}(\omega)\right]}\right\rangle
$$

The first term is also present for the phase-conjugating mirror, so we only need to consider the second term. This term can be written as

$$
\begin{aligned}
\left\langle\frac{t^{2}(\omega+\delta \omega) t^{2 *}(\omega)}{[1+r(\omega+\delta \omega)]\left[1+r^{*}(\omega)\right]}\right\rangle & =\sum_{n, m}(-1)^{n+m}\left\langle t^{2}(\omega+\delta \omega) t^{2 *}(\omega) r^{n}(\omega+\delta \omega) r^{m *}(\omega)\right\rangle \\
& =\sum_{n}\left\langle t^{2}(\omega+\delta \omega) t^{2 *}(\omega) r^{n}(\omega+\delta \omega) r^{n *}(\omega)\right\rangle,
\end{aligned}
$$

where we have averaged over $\theta$ in the last step.

Now we consider the phase-conjugating mirror in the regime $\tau_{s} \omega \gg 1$. In that regime the phase $\theta(\omega)$ is independent of the phase $\theta(-\omega)$. The power spectrum $a(\omega, \delta \omega)$ can again be written in terms of $R, \theta, \theta^{\prime}$ [Eqs. (8) and (15)]. Only three terms survive the average over $\theta( \pm \omega), \theta^{\prime}(\omega)$, 


$$
\begin{aligned}
4\langle a(\omega, \delta \omega)\rangle= & \left\langle r^{\prime}(\omega+\delta \omega) r^{*}(\omega)\right\rangle+\left\langle\frac{t^{2}(\omega+\delta \omega) r^{*}(-\omega-\delta \omega) t^{2 *}(\omega) r(-\omega)}{\left[1-r^{*}(-\omega-\delta \omega) r(\omega+\delta \omega)\right]\left[1-r(-\omega) r^{*}(\omega)\right]}\right\rangle \\
& +\left\langle\frac{t(\omega+\delta \omega) t^{*}(-\omega-\delta \omega) t^{*}(\omega) t(-\omega)}{\left[1-r^{*}(-\omega-\delta \omega) r(\omega+\delta \omega)\right]\left[1-r(-\omega) r^{*}(\omega)\right]}\right\rangle .
\end{aligned}
$$

The first term is also present for the normal mirror. For $\tau_{s} \omega \gg 1, t(\omega)$ is independent of $t(-\omega)$. The second term is then much larger than the third term because of the large fluctuations in the localized regime $(L \gg l)$. The second term can also be written as

$$
\begin{aligned}
& \left\langle\frac{t^{2}(\omega+\delta \omega) r^{*}(-\omega-\delta \omega) t^{2 *}(\omega) r(-\omega)}{\left[1-r^{*}(-\omega-\delta \omega) r(\omega+\delta \omega)\right]\left[1-r(-\omega) r^{*}(\omega)\right]}\right\rangle \\
& =\sum_{n, m}\left\langle t^{2}(\omega+\delta \omega) t^{2 *}(\omega) r^{n}(\omega+\delta \omega) r^{m *}(\omega) r^{m+1}(-\omega) r^{n+1 *}(-\omega-\delta \omega)\right\rangle \\
& =\sum_{n}\left\langle t^{2}(\omega+\delta \omega) t^{2 *}(\omega) r^{n}(\omega+\delta \omega) r^{n *}(\omega)\right\rangle\left\langle r^{n+1}(-\omega) r^{n+1 *}(-\omega-\delta \omega)\right\rangle .
\end{aligned}
$$

Comparison with Eq. (B2) for a normal mirror shows that the two expressions are the same as long as we can replace $\left\langle r^{n+1}(-\omega) r^{n+1 *}(-\omega-\delta \omega)\right\rangle$ by 1 for the relevant terms in the summation over $n$. It is now convenient to write $r^{n}(\omega$ $+\delta \omega) r^{n *}(\omega)=R^{n}(\omega)[1-C(\omega, \delta \omega)]^{n}$. The average over $\{r(\omega), r(\omega+\delta \omega), t(\omega), t(\omega+\delta \omega)\}$ is dominated by configurations where the transmittance $T$ is large. For small $\delta \omega$ this corresponds to configurations where $1-R(\omega)$ and $|C(\omega, \delta \omega)|$ are much larger than typical values of these quantities. For these dominating configurations the number of relevant terms in the summation over $n$ is relatively small and for these relatively small $n$ we can replace $\left\langle r^{n+1}(-\omega) r^{n+1 *}(-\omega-\delta \omega)\right\rangle$ by 1 . We therefore conclude that for small $\delta \omega$, the average power spectrum $\langle a(\omega, \delta \omega)\rangle$ is the same as for a normal mirror. The above argument breaks down if $\left\langle r^{n+1}(-\omega) r^{n+1 *}(-\omega-\delta \omega)\right\rangle$ starts to deviate from 1 for the relevant terms in the summation. This is the case for $\ln \left(1 / \tau_{s} \delta \omega\right) \lesssim L / l$.

${ }^{1}$ Scattering and Localization of Classical Waves in Random Media, edited by P. Sheng (World Scientific, Singapore, 1990).

${ }^{2}$ M. Asch, W. Kohler, G. Papanicolaou, M. Postel, and B. White, SIAM Rev. 33, 519 (1991).

${ }^{3}$ Diffuse Waves in Complex Media, edited by J.-P. Fouque, NATO Science Series C531 (Kluwer, Dordrecht, 1999).

${ }^{4}$ C. W. J. Beenakker, in Photonic Crystals and Light Localization in the 21st Century, edited by C. M. Soukoulis, NATO Science Series C563 (Kluwer, Dordrecht, 2001).

${ }^{5}$ B. White, P. Sheng, Z. Q. Zhang, and G. Papanicolaou, Phys. Rev. Lett. 59, 1918 (1987).

${ }^{6}$ M. Titov and C. W. J. Beenakker, Phys. Rev. Lett. 85, 3388 (2000).

${ }^{7}$ B. L. Altshuler and V. N. Prigodin, JETP Lett. 47, 43 (1988).

${ }^{8}$ B. L. Altshuler, V. E. Kravtsov, and I. V. Lerner, in Mesoscopic Phenomena in Solids, edited by B. L. Altshuler, P. A. Lee, and R. A. Webb (North-Holland, Amsterdam, 1991).

${ }^{9}$ B. A. Muzykantskii and D. E. Khmelnitskii, Phys. Rev. B 51, 5480 (1995).

${ }^{10}$ C. J. Bolton-Heaton, C. J. Lambert, V. I. Falko, V. Prigodin, and A. J. Epstein, Phys. Rev. B 60, 10569 (1999).

${ }^{11}$ A. D. Mirlin, Phys. Rep. 326, 259 (2000).

${ }^{12}$ Optical Phase Conjugation, edited by R. A. Fisher (Academic, New York, 1983).
${ }^{13}$ B. Ya. Zeldovich, N. F. Pilipetskii, and V. V. Shkunov, Principles of Phase Conjugation (Springer, Berlin, 1985).

${ }^{14}$ V. I. Yudson and P. Reineker, Phys. Rev. B 45, 2073 (1992).

${ }^{15}$ J. C. J. Paasschens, M. J. M. de Jong, P. W. Brouwer, and C. W. J. Beenakker, Phys. Rev. A 56, 4216 (1997).

${ }^{16}$ M. Blaauboer, D. Lenstra, and A. Lodder, Superlattices Microstruct. 23, 937 (1998).

${ }^{17}$ P. W. Brouwer, K. M. Frahm, and C. W. J. Beenakker, Waves Random Media 9, 91 (1999).

${ }^{18}$ V. L. Berezinskii, Sov. Phys. JETP 38, 620 (1974).

${ }^{19}$ V. Freilikher, M. Pustilnik, and I. Yurkevich, Phys. Rev. Lett. 73, 810 (1994).

${ }^{20}$ H. U. Baranger, D. P. DiVincenzo, R. A. Jalabert, and A. D. Stone, Phys. Rev. B 44, 10637 (1991).

${ }^{21}$ B. L. Altshuler and V. N. Prigodin, Sov. Phys. JETP 68, 198 (1989).

${ }^{22}$ V. L. Berezinskii and L. P. Gorkov, Sov. Phys. JETP 50, 1209 (1979).

${ }^{23}$ J. Gómez Rivas, R. Sprik, A. Lagendijk, L. D. Noordam, and C. W. Rella, Phys. Rev. E 63, 046613 (2001).

${ }^{24}$ A. A. Chabanov and A. Z. Genack, Phys. Rev. Lett. 87, 233903 (2001).

${ }^{25}$ V. I. Melnikov, Sov. Phys. Solid State 22, 1398 (1980). 